\title{
A Cost-Utility Analysis of Lisdexamfetamine Versus Atomoxetine in the Treatment of Children and Adolescents with Attention- Deficit/Hyperactivity Disorder and Inadequate Response to Methylphenidate
}

\author{
Evelina A. Zimovetz ${ }^{1} \cdot$ Stephen M. Beard $^{2} \cdot$ Paul Hodgkins $^{3} \cdot$ Matthias Bischof $^{4}$. \\ Josephine A. Mauskopf ${ }^{5} \cdot$ Juliana Setyawan $^{3}$
}

Published online: 16 August 2016

(C) The Author(s) 2016. This article is published with open access at Springerlink.com

\begin{abstract}
Background An economic analysis from the perspective of the UK National Health Service (NHS) evaluated the cost effectiveness of lisdexamfetamine dimesylate (LDX) compared with atomoxetine in children and adolescents with attention-deficit/hyperactivity disorder who have had an inadequate response to methylphenidate.

Methods A 1-year decision-analytic model was constructed, with the health outcomes "response", "nonresponse", and "unable to tolerate". Clinical data were taken from a head-to-head, randomized controlled trial in inadequate responders to methylphenidate. Response to treatment was defined as a score of 1 (very much improved) or 2 (much improved) on the Clinical Global ImpressionImprovement subscale. Tolerability was assessed by discontinuation rates owing to adverse events. Utility weights
\end{abstract}

Electronic supplementary material The online version of this article (doi:10.1007/s40263-016-0354-3) contains supplementary material, which is available to authorized users.

S. M. Beard, P. Hodgkins, M. Bischof, J. A. Mauskopf, and J. Setyawan contributed equally to this work.

Evelina A. Zimovetz

ezimovetz@rti.org

Stephen M. Beard

smbeard@btinternet.com

Paul Hodgkins

paulhodgkins@verizon.net

Matthias Bischof

mbischof@shire.com

Josephine A. Mauskopf

jmauskopf@rti.org

Juliana Setyawan

juliana.setyawan@gmail.com were identified via a systematic literature review. Healthcare resource use estimates were obtained via a survey of clinicians. Daily drug costs were derived from British National Formulary 2012 costs and mean doses reported in the trial. One-way and probabilistic sensitivity analyses (PSAs) were performed.

Results The comparison of LDX with atomoxetine resulted in an estimate of an incremental cost-effectiveness ratio of $£ 1802$ per quality-adjusted life-year (QALY). The result was robust in a wide range of sensitivity analyses; results were most sensitive to changes in drug costs and efficacy. In the PSA, assuming a maximum willingness to pay of $£ 20,000$ per QALY, LDX versus atomoxetine had an $86 \%$ probability of being cost effective. In $38 \%$ of PSA runs, LDX was more effective and less costly than atomoxetine. Conclusions From the perspective of the UK NHS, LDX provides a cost-effective treatment option for children and adolescents who are inadequate responders to methylphenidate.

Market Access and Outcomes Strategy, RTI Health Solutions, 2nd Floor, The Pavilion, Towers Business Park, Wilmslow Road, Didsbury, Manchester M20 2LS, UK

2 RTI Health Solutions, Sheffield, UK

3 Global HEOR and Epidemiology, Shire, Wayne, PA, USA

4 Global HEOR and Epidemiology, Shire, Zug, Switzerland

5 RTI Health Solutions, Research Triangle Park, NC, USA 


\section{Key Points}

About one-third of patients have an inadequate response to initial treatment with methylphenidate and consequently might be considered for treatment with atomoxetine or lisdexamfetamine dimesylate (LDX).

In this patient population, LDX was associated with a faster and more robust treatment response than atomoxetine, as demonstrated in a 9-week, head-tohead, randomized, double-blind, active-controlled study.

An economic analysis, conducted using data from the head-to-head trial, showed that LDX is a costeffective treatment option compared with atomoxetine in children and adolescents who have had an inadequate response to methylphenidate.

\section{Introduction}

Attention-deficit/hyperactivity disorder (ADHD) is a chronic neurobehavioral disorder that is estimated to affect $5.3 \%$ of children worldwide, $5 \%$ of European children, and $6-6.5 \%$ of children in North America $[1,2]$. The core symptoms of ADHD include inattention, impulsivity, and hyperactivity [3]. It is associated with impairment that affects not only the child but also parents and siblings, causing disturbances to family and marital functioning, and reduction in the overall quality of life [4, 5]. ADHD often persists into adulthood, causing disruptions to both professional and personal life [4] and imposes a considerable cost on the healthcare system and society. In the USA, the economic burden of ADHD in children and adolescents is estimated to be between \$US38 billion and \$US72 billion per year, of which healthcare cost estimates range between \$US21 billion and \$US44 billion [6]. A review of European-based studies of ADHD-related costs estimated annual average total costs to be between $€ 9860$ and $€ 14,483$ per patient [7]. In Europe, the annual national costs were estimated to be between $€ 1041$ and $€ 1529$ million [7], using the Netherlands as a reference country.

Management of ADHD usually includes psychotherapy and behavioral therapy, parent training, medications, or a combination of both psychotherapy and pharmacotherapy. In the UK, the stimulant methylphenidate is a recommended first-line treatment, with the nonstimulant atomoxetine considered for first-line treatment when tics, Tourette's syndrome, anxiety disorder, stimulant misuse, or risk of stimulant diversion are present [8]. It has been estimated that as many as $25-35 \%$ of subjects in clinical trials may have an inadequate response to initial stimulant treatment [9]. In the UK, atomoxetine is the recommended treatment option in patients who have had inadequate response or are intolerant to methylphenidate [8, 10]. Atomoxetine was found to be a cost-effective option compared with stimulant therapy (represented in the model by methylphenidate and dexamphetamine) when evaluated from the perspective of the National Health Service (NHS) in the stimulant-exposed UK population [11].

Lisdexamfetamine dimesylate (LDX) is a pro-drug; following absorption, LDX undergoes hydrolysis to dexamphetamine. LDX has been studied in several large, phase III trials in children and adolescents and has been shown to be effective in reducing the symptoms of ADHD. Three trials in children and adolescents with ADHD were placebo controlled [12-14], and one was a head-to-head study versus atomoxetine in children and adolescents with ADHD who have had an inadequate response to methylphenidate (either immediate or extended release) [15]. In the head-to-head study, the median time to first clinical response, defined as a Clinical Global Impressions-Improvement (CGI-I) score of 1 (very much improved) or 2 (much improved), $95 \%$ confidence interval (CI) was significantly shorter for patients receiving LDX (12.0 days [8.0-16.0]) than for those receiving atomoxetine (21.0 days [15.0-23.0]) ( $p=0.001)$ [15]. Moreover, at the completion of the 9-week study period, a greater proportion of patients achieved improved global functioning, as measured by the CGI-I [16], designed to rate severity of illness and improvement in symptom severity (103/126 patients [81.7\%] in the LDX group compared with 84/132 patients [63.6\%] in the atomoxetine group) [15]. Similarly, a greater proportion of patients receiving LDX experienced improvement in symptoms, as measured by the ADHD Rating Scale-IV, designed to reflect current symptomatology of ADHD based on Diagnostic and Statistical Manual of Mental Disorders, 4th edition, text revision (DSM-IV-TR) criteria [17] (114/126 patients [90.5\%] in the LDX group achieved response [defined as a percentage reduction from baseline in the ADHD-RS-IV total score of $\geq 25 \%$ ] compared with $102 / 133$ patients [76.7\%] in the atomoxetine group) [18]. Although estimates of the time required for atomoxetine to reach its maximum effect generally range from 4 to 6 weeks $[8,19]$, one study suggests that it may take as long as 12 weeks [20]. The objective of the present study is to estimate the cost effectiveness of LDX compared with atomoxetine in the treatment of children and adolescents with ADHD in whom response to methylphenidate (either extended release or immediate release) is considered clinically inadequate. The results of the analysis are presented as the total costs and total quality-adjusted life-years (QALYs) 
for each drug, as well as the incremental costs and QALYs for LDX when compared with atomoxetine. The incremental cost-effectiveness ratio (ICER) for LDX relative to atomoxetine is also presented and evaluated against an established cost-effectiveness threshold of $£ 20,000$ per QALY [21].

\section{Methods}

A decision-tree model was developed in Microsoft ${ }^{\circledR}$ Excel (Microsoft Corp., Redmond, WA, USA) to evaluate the cost effectiveness of LDX compared with atomoxetine from the perspective of the UK NHS. The health outcomes used in the model as the measures of benefit were "unable to tolerate", "response", and "nonresponse" (Fig. 1). The impact of using LDX as an alternative to atomoxetine in terms of costs and outcomes was estimated in the model by simulating the number of patients who achieved a response to treatment and those who did not. Costs and utilities corresponding to the patients' health states were assigned to each of these patients. The target population for the analysis consisted of children and adolescents with ADHD in whom response to methylphenidate was considered clinically inadequate. An inadequate response to methylphenidate, as defined in the LDX head-to-head study versus atomoxetine [15] included, but was not limited to, one or more of the following: the presence of some residual ADHD symptoms; inadequate duration of action; variable symptom control; as well as whether, in the opinion of the investigator, the patient may benefit from an alternative to methylphenidate [15]. The base-case analysis evaluated direct medical costs and health-related quality of life associated with 1 year of treatment, including the initial 28-day drug titration period. The time horizon of 1 year was chosen to capture differences in the cost and benefits for the time period of the clinical trial, given the lack of long-term data comparing LDX and atomoxetine. The dichotomous response modelling framework and key assumptions, including the 1-year time horizon, were adapted from the health technology assessment (HTA) model used in an earlier assessment of ADHD drugs by the UK National Institute for Health and Care Excellence (NICE) [22].

\subsection{Model Assumptions}

Patients who inadequately responded to methylphenidate entered the model when they initiated a course of treatment with either LDX or atomoxetine.

Patients who experienced intolerable side effects discontinued treatment in the middle of the titration period, i.e., after 14 days of treatment. The discontinuation of patients' utilities and costs during the titration period (28 days) was represented by a 50/50 \% mix of the responder and nonresponder utility values and a 50/50 \% mix of the responder and nonresponder nondrug costs, respectively.

Patients who discontinued treatment because of intolerable side effects did not initiate additional treatment because they have failed both methylphenidate and a second-line drug. Patients who discontinued because of intolerable side effects had the same utilities and costs as nonresponders for the remainder of the 1-year model time horizon.

Patients who responded to treatment at the end of the titration period continued to receive treatment throughout the model's time horizon, maintaining their level of response.

At the end of the titration period, nonresponding patients discontinued all drug treatments and were assumed to stay in the nonresponse health state for the remainder of the model time horizon. Patients who discontinued because of
Fig. 1 Model structure. $A D H D$ attention-deficit/hyperactivity disorder, $A T X$ atomoxetine, $L D X$ lisdexamfetamine dimesylate, $M P H$ methylphenidate, $N T$ no treatment

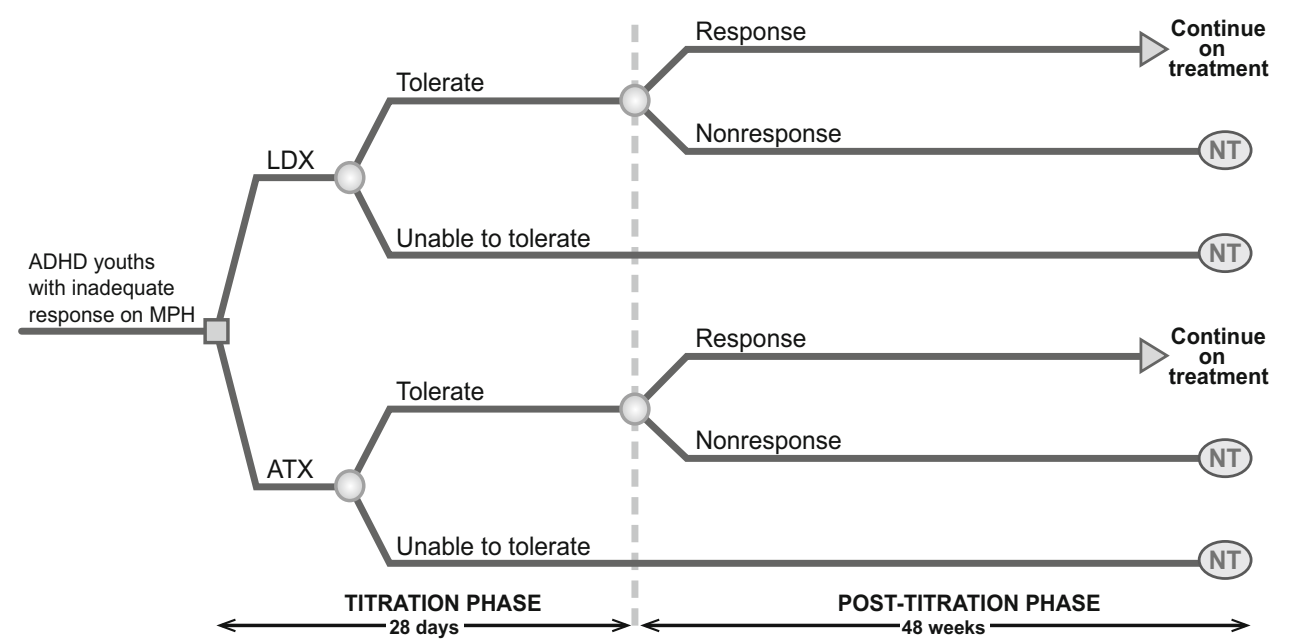


lack of response had immediate loss of treatment effect and were assigned the associated costs and utilities throughout the model's time horizon.

The analysis compares LDX and atomoxetine administered as directed in the head-to-head study [15]. We assumed that patients who responded and tolerated treatment were adherent and persistent with treatment over the time horizon of the model, as was generally observed in the head-to-head study. This assumption is consistent with that made in the HTA model presented by King et al. [22].

Costs and benefits were not discounted, given the time horizon of 1 year. The uncertainty in the ICER estimate was explored by one-way and probabilistic sensitivity analyses (PSAs).

Quality control of model programming and verification of all input data with original sources was performed according to a prespecified test plan by health economists who were not involved in the model development.

\subsection{Model Parameter Inputs}

\subsubsection{Efficacy and Safety}

The base-case analysis used data generated from a head-tohead clinical study that compared LDX versus atomoxetine, the most commonly used second-line treatment in the UK in patients with ADHD who were inadequate responders to previous methylphenidate therapy [15]. Head-tohead randomized controlled trials are considered by NICE to provide the most valid evidence of relative efficacy; hence, this study was used in the economic analysis. That study was a 9-week, head-to-head, randomized, doubleblind, active-controlled study (SPD489-317; ClinicalTrials.gov NCT01106430), with patients randomized 1:1 to an optimized daily dose of $\operatorname{LDX}(30,50$, or $70 \mathrm{mg})$ or atomoxetine (patients $<70 \mathrm{~kg}, 0.5-1.2 \mathrm{mg} / \mathrm{kg}$ with total daily dose not exceeding $1.4 \mathrm{mg} / \mathrm{kg}$; patients $\geq 70 \mathrm{~kg}, 40,80$, or $100 \mathrm{mg}$ ). Response to treatment, assumed in the model to be measured at the end of the titration period, was defined as a score of 1 (very much improved) or 2 (much improved), based on the last observation carried forward (LOCF) CGI-I score at week 9, which was the definition of response used in the primary outcome. This definition was also used in the trial to categorize patients into responders and nonresponders, and this outcome was selected for use in the economic analysis owing to its dichotomous nature. Response rates applied in the base-case analysis are presented in Table 1.

Similar proportions of patients in the LDX and atomoxetine groups experienced treatment-emergent adverse events: 92/128 (72\%) and 95/134 (71\%), respectively [15]. Therefore, short-term drug-related adverse events were not incorporated in the model. Patients' drug tolerability in the model was based on the rates of withdrawal because of adverse events reported in the head-tohead trial of LDX versus atomoxetine (6.3 and $7.5 \%$, respectively) [15].

\subsubsection{Health-State Utilities}

A systematic review of the economic literature in ADHD was conducted to identify utility values for inclusion in the model (methods of this review are reported elsewhere [31]). Utility estimates associated with response and nonresponse from the study by Coghill et al. [24] were selected as relevant for the economic analysis (Table 1). These values were estimated using the EuroQol-5 Dimensions instrument (EQ-5D), which is NICE's preferred method for obtaining utility values [21].

\subsubsection{Resource Use and Costs}

Healthcare resource utilization estimates associated with response and nonresponse were obtained from a survey of clinicians currently treating patients with ADHD in the UK [27] (Table 2). The sample consisted of 21 specialists (18 from England, two from Scotland, and one from Wales); 13 of the specialists were psychiatrists, the remaining eight were pediatricians. Unit costs from 2011-2012 UK national sources were applied to the resource utilization estimates to calculate the costs associated with nondrug healthcare resources used by responders and nonresponders. In the survey, items of healthcare resource were based on those reported by King et al. [22].

Drug unit costs were obtained from the British National Formulary 2012 [29]. Drug doses used were the mean doses for the titration and maintenance periods as reported in the head-to-head trial. Drug costs were calculated using the head-to-head trial mean doses and permilligram drug costs. Each per-milligram cost was based on the cost of a pack with the tablet size closest to the given mean dose.

\subsection{Sensitivity Analyses}

\subsubsection{One-Way Sensitivity Analysis}

The following summarizes the variables considered in the one-way sensitivity analysis (Table 1).

Response rates The base-case analysis used a definition of treatment response based on the LOCF CGI-I measure at week 9. A sensitivity analysis applied response rates based on the ADHD Rating Scale-IV measure, using a reduction of $25 \%$ or greater in ADHD Rating Scale-IV score (the most commonly used threshold in ADHD trials [23]), assessed using the result at week 9 or a non-response for 
Table 1 Summary of model input parameters

\begin{tabular}{|c|c|c|c|c|c|}
\hline Description & Analysis ${ }^{\mathrm{a}}$ & Value & Range & Distribution & Source \\
\hline \multicolumn{6}{|c|}{ Treatment-specific response rates } \\
\hline \multicolumn{6}{|c|}{ Response defined as the proportion of patients with a CGI-I value of 1 (very much improved) or 2 (much improved) out of all patients included } \\
\hline LDX & Base & $81.7 \%$ & $75.0-88.5 \%$ & Beta & [15] \\
\hline ATX & Base & $63.6 \%$ & $55.4-71.8 \%$ & Beta & {$[15]$} \\
\hline LDX & SA & $\begin{array}{l}70.3 \% \\
R R=3.218\end{array}$ & $2.683-3.860$ & Log-normal $^{\mathrm{c}}$ & MTC [23] \\
\hline ATX & SA & $\begin{array}{l}45.4 \% \\
\mathrm{RR}=2.077\end{array}$ & $1.782-2.419$ & Log-normal ${ }^{c}$ & MTC [23] \\
\hline
\end{tabular}

Response defined as the proportion of patients with a percentage reduction from baseline in ADHD-RS-IV total score of $\geq 25 \%{ }^{\mathrm{d}}$

$\begin{array}{llllll}\text { LDX } & \text { SA } & 72.4 \% & 64.7-80.2 \% & \text { Beta } & \text { Head-to-head trial data [18] } \\ \text { ATX } & \text { SA } & 63.7 \% & 55.6-71.8 \% & \text { Beta } & \text { Head-to-head trial data [18] }\end{array}$

Treatment-specific withdrawal owing to AE rates

$\begin{array}{llllll}\text { LDX } & \text { Base } & 6.25 \% & \text { NR } & \text { Beta } & \text { [15] } \\ \text { ATX } & \text { Base } & 7.5 \% & \text { NR } & \text { Beta } & \text { [15] } \\ \text { LDX } & \text { SA } & 3 \% & 0.942-8.755 & \text { Log-normal }^{\text {c }} & \text { MTC [23] } \\ & & \text { RR }=2.871 & & & \\ \text { ATX } & \text { SA } & 1.7 \% & 0.836-3.331 & \text { Log-normal }^{\mathrm{c}} & \text { MTC [23] } \\ & & \text { RR }=1.669 & & & \end{array}$

Health-state utilities

\begin{tabular}{|c|c|c|c|c|c|}
\hline Responder & Base & 0.837 & NR & Beta & EQ-5D [24] \\
\hline Nonresponder & Base & 0.773 & NR & Beta & EQ-5D [24] \\
\hline Responder & SA & 0.820 & $\mathrm{SE}=0.19$ & Beta & HUI2 [25] \\
\hline Nonresponder & SA & 0.700 & $\mathrm{SE}=0.20$ & Beta & HUI2 [25] \\
\hline Responder & SA & 0.926 & $0.915-0.938$ & Beta & Head-to-head trial data analysis [26] \\
\hline Nonresponder & SA & 0.905 & $0.886-0.924$ & Beta & Head-to-head trial data analysis [26] \\
\hline \multicolumn{6}{|c|}{ Health-state costs (each 28 days) } \\
\hline Responder & Base & $£ 99.78$ & $£ 78.82-119.74$ & $\begin{array}{l}\text { Uniform } \\
\qquad( \pm 20 \%)\end{array}$ & $\begin{array}{l}\text { Resource use estimates [27] } \\
\text { Unit costs [28] }\end{array}$ \\
\hline Nonresponder & Base & $£ 190.25$ & NA & $\begin{array}{l}\text { Uniform } \\
\qquad( \pm 20 \%)\end{array}$ & $\begin{array}{l}\text { Resource use estimates [27] } \\
\text { Unit costs [28] }\end{array}$ \\
\hline Responder & SA & $£ 109.41$ & NA & $\begin{array}{l}\text { Uniform } \\
\qquad( \pm 20 \%)\end{array}$ & $\begin{array}{l}\text { Estimates [22] } \\
\text { Unit costs [28] }\end{array}$ \\
\hline Nonresponder & SA & $£ 162.78$ & NA & $\begin{array}{l}\text { Uniform } \\
( \pm 20 \%)\end{array}$ & $\begin{array}{l}\text { Estimates [22] } \\
\text { Unit costs [28] }\end{array}$ \\
\hline Responder & SA & $\begin{array}{l}£ 93.38, £ 80.57 \\
£ 67.76\end{array}$ & Not included & Not included & $\begin{array}{l}-10 \%,-30 \%,-50 \% \text { specialist visit vs. junior doctor } \\
\text { assumption }\end{array}$ \\
\hline Nonresponder & SA & $\begin{array}{l}£ 177.59, £ 152.26, \\
£ 126.94\end{array}$ & Not included & Not included & $\begin{array}{l}-10 \%,-30 \%,-50 \% \text { specialist visit vs. junior doctor } \\
\text { assumption }\end{array}$ \\
\hline \multicolumn{6}{|c|}{ Drug costs (per 28 days) } \\
\hline LDX & Base & $£ 83.02$ (titration) & Not included & Not included & Usage [18] \\
\hline & & $\begin{array}{l}£ 72.28 \text { (post } \\
{\text { titration })^{\mathrm{f}}}\end{array}$ & & & Unit costs [29] \\
\hline ATX & Base & $£ 82.43$ (titration) & Not included & Not included & Usage [18] \\
\hline & & $\begin{array}{l}£ 63.03(\text { post } \\
{\text { titration })^{\mathrm{f}}}\end{array}$ & & & Unit costs [29] \\
\hline
\end{tabular}


Table 1 continued

\begin{tabular}{|c|c|c|c|c|c|}
\hline Description & Analysis $^{a}$ & Value & Range & Distribution & Source \\
\hline LDX & SA & $\begin{array}{l}£ 83.02 \text { (titration) } \\
£ 66.56 \text { (post } \\
\text { titration) }^{\mathrm{g}}\end{array}$ & Not included & Not included & $\begin{array}{l}\text { Usage [30] } \\
\text { Unit costs [29] }\end{array}$ \\
\hline ATX & SA & $\begin{array}{l}£ 82.43 \text { (titration) } \\
£ 107.77 \text { (post } \\
\text { titration) }^{\mathrm{g}}\end{array}$ & Not included & Not included & $\begin{array}{l}\text { Usage [30] } \\
\text { Unit costs [29] }\end{array}$ \\
\hline
\end{tabular}

ADHD-RS-IV Attention-Deficit/Hyperactivity Disorder Rating Scale-IV, AE adverse event, $A T X$ atomoxetine, CGI-I Clinical Global ImpressionImprovement subscale, EQ-5D EuroQol 5-Dimensions instrument, HUI2 Health Utilities Index Mark 2, LDX lisdexamfetamine dimesylate, MTC mixed-treatment comparison, $N A$ not applicable, $N R$ not reported, $P S A$ probabilistic sensitivity analysis, $R R$ relative risk, $S A$ sensitivity analysis, $S E$ standard error

${ }^{a}$ Base-case analysis or sensitivity analysis

b Assessed as the last observation carried forward at week 9

c The MTC outputs for response and discontinuation because of AEs were provided as RRs versus placebo. These RRs were converted in the economic model to rates using the MTC pooled placebo rates ( 0.218 for response and 0.010 for withdrawals). To generate PSA values, sampling was performed on RRs using log-normal distributions

d Assessed using the result at week 9 or a non-response for those not assessed at week 9 (a non-response imputation approach to dealing with missing data)

e This proportion of visits to a specialist (psychiatrist or pediatrician) was costed using the unit cost for a visit to a junior doctor (foundation house officer 1 at $£ 43$ per $\mathrm{h}$ ) rather than as a visit to a consultant

${ }^{f}$ This method of drug costing uses the mean doses from the head-to-head trial. In calculating the daily drug costs applied in the model, the mean daily doses for titration and post-titration periods were multiplied by the per-milligram costs. Each per-milligram cost was based on the cost of a package with a tablet size closest to the given mean daily dose. Per-cycle cost was based on a daily cost multiplied by 28 days

$\mathrm{g}$ This method of drug costing was derived from the average daily number of tablets consumed, as reported in the IMS database [30]. To calculate daily drug costs, the daily number of tablets consumed, split by tablet strength, was multiplied by the corresponding tablets' unit costs and then weighted by the corresponding prescription volumes. The estimates of daily consumption and the prescription shares for LDX's different tablet strengths were based on the IMS data collected in Brazil and Canada (where LDX has been used in clinical practice)

those not assessed at week 9 (non-responder imputation [NRI] approach). The NRI-based value for ADHD-RS score rather than the LOCF-based value was chosen to explore how the cost-effectiveness results would change when the LDX response rate decreased while the atomoxetine rate remained roughly the same. A further sensitivity analysis used response rates from a mixedtreatment comparison (MTC) analysis conducted using ADHD clinical trials in a broad ADHD population (rather than in patients with inadequate response to methylphenidate who represented the model target population) (Table 1). Despite this difference in the populations, the MTC results were used in the sensitivity analysis to explore the impact of utilizing all published clinical evidence in the model. Systematic literature review and MTC methods used are presented in the Electronic Supplementary Material (ESM) Resource 1 and 2, with further details reported elsewhere [23]; the search strategy is presented in ESM Resource 3.

Utility values In the base-case analysis, the model applied health-state utility estimates reported by Coghill et al. [24], who used the EQ-5D instrument to obtain utility values. The sensitivity analysis applied alternative utility values reported by Lloyd et al. [25], who used statistical mapping to derive utilities for responder and nonresponder health states defined using the CGI-I measure. A further sensitivity analysis applied utility data from the LDX versus atomoxetine head-to-head trial in patients who had an inadequate response to methylphenidate (Table 1) [26]. These utility values were considered particularly valuable as they were derived from the population studied in the head-to-head trial. Patients in that study were evaluated using the Health Utilities Index Mark 2 (HUI2) [33]. The selfadministered, proxy-assessed version of the HUI2 consists of 15 questions used to classify a patient's health status. In the trial, the proxy was the patient's parent or legally authorized representative.

Resource use estimates The base-case analysis used the healthcare resource use estimates obtained from a survey of UK specialists. In the sensitivity analysis, these resource use estimates were replaced by the values reported in the HTA model [22]. To calculate the costs, the 2011-2012 UK resource unit costs were applied to these alternative values. In additional sensitivity analyses, a percentage of the visits to specialists were assumed to be consultations 
Table 2 Resource use and costs applied in the base-case analysis

\begin{tabular}{llll}
\hline Resource item & Unit cost & Units per year & Average cost per year \\
\hline Responders & & & \\
Psychiatrist $^{\mathrm{a}}$ & $£ 261.87$ & 2.48 & $£ 649.44$ \\
Pediatrician $^{\mathrm{b}}$ & $£ 167.41$ & 2.33 & $£ 390.07$ \\
GP $^{\mathrm{c}}$ & $£ 43.00$ & 2.62 & $£ 112.66$ \\
Nurse $^{\mathrm{d}}$ & $£ 49.00$ & 2.71 & $£ 132.79$ \\
Blood test $^{\mathrm{e}}$ & $£ 3.09$ & 0.42 & $£ 1.30$ \\
ECG $^{\mathrm{f}}$ & $£ 60.73$ & 0.18 & $£ 10.93$ \\
Total $^{\text {Nonresponders }}$ & - & - & $£ 1297$ \\
Psychiatrist $^{\mathrm{a}}$ & $£ 261.87$ & 5.19 & \\
Pediatrician $^{\mathrm{b}}$ & $£ 167.41$ & 4.1 & $£ 1359.11$ \\
GP $^{\mathrm{c}}$ & $£ 43.00$ & 4.24 & $£ 686.38$ \\
Nurse $^{\mathrm{d}}$ & $£ 49.00$ & 4.48 & $£ 182.32$ \\
Blood test $^{\mathrm{e}}$ & $£ 3.09$ & 0.72 & $£ 219.52$ \\
ECG $^{\mathrm{f}}$ & $£ 60.73$ & 0.39 & $£ 2.22$ \\
Total & - & - & $£ 23.68$ \\
\hline
\end{tabular}

$E C G$ electrocardiogram, GP general practitioner, NHS National Health Service

${ }^{a}$ Source: NHS Trusts and NHS Foundation Trusts: Mental HealthOutpatient Attendances. MHOP05-Children and Adolescent Other Services [28]

b Source: NHS Trusts and NHS Foundation Trusts: Consultant LedFollow up Attendance Non-Admitted Face to Face. 420_-Paediatrics [28]

c Source: $10.8 \mathrm{~b}$ General practitioner-unit costs. Per surgery consultation lasting $11.7 \mathrm{~min}$ [32]

${ }^{\mathrm{d}}$ Source: 10.4 Nurse specialist [community]—unit costs. Per h [32]

e Source: NHS Trusts and NHS Foundation Trusts: Direct AccessPathology Services. DAP823-Haematology [Excluding Anti-Coagulant Services] [28]

${ }^{\mathrm{f}}$ Source: NHS Trusts and NHS Foundation Trusts: Direct AccessDiagnostic Services. EA47Z-Electrocardiogram Monitoring and Stress Testing [28]

with junior doctors and were costed accordingly. Three analyses were performed by replacing 10,30 , and $50 \%$ of specialist visits to pediatricians or psychiatrists with visits to junior doctors.

Drug-costing method The base-case analysis used a method of drug costing whereby the mean daily doses from the trial were used to calculate the daily drug costs. The sensitivity analysis explored the effect of applying the drug costs calculated from real-world drug utilization, rather than from drug usage reported in the trial. The realworld usage data were obtained from Canada and Brazil, where LDX has been used in clinical practice. These data were reported as the daily average consumption, presented by the numbers of tablets [30]. To calculate the corresponding costs, the tablet numbers were multiplied by tablet unit costs.

\subsubsection{Probabilistic Sensitivity Analysis}

Uncertainty in model input parameters was examined in PSAs, wherein all input parameters, apart from drug costs, which were known with certainty, were simultaneously varied using prespecified distributions reflecting the uncertainty about their true values. The prespecified distributions for each parameter included in the PSA are presented in Table 1; 1000 repeated model simulations were performed. The PSA was performed using the basecase input parameter values and using two alternative scenarios: one using efficacy distributions estimated from the MTC, and one using utility weight distributions calculated from the head-to-head trial data. PSA results for the base-case analysis were presented graphically in the form of a cost-effectiveness acceptability curve.

\section{Results}

\subsection{Base-Case Analysis}

The base-case analysis results are presented in Table 3 . Over a time horizon of 1 year, a patient receiving LDX benefitted from 0.011 additional QALYs compared with a patient receiving atomoxetine. The estimated mean perpatient drug cost was $£ 745$ in the LDX arm and $£ 525$ in the atomoxetine arm; a difference of $£ 220$ between arms. The per-patient nondrug cost was $£ 1607$ and $£ 1807$ for patients receiving LDX and atomoxetine, respectively; thus, total costs were $£ 2352$ for LDX and $£ 2332$ for atomoxetine. The ICER of using LDX versus atomoxetine was estimated as $(£ 2352-2332) / 0.011=£ 1802$ per QALY (note: calculations used unrounded values).

\subsection{One-Way Sensitivity Analysis}

The results of the one-way sensitivity analyses are summarized in Fig. 2 and Table 4. The results of the model were found to be robust to changes in the majority of the model input parameter values. The model was most sensitive to changes in assumptions about drug costs and to the use of MTC-generated response rates, with the direction of results changing from LDX being cost effective in the base case to LDX being dominant-that is, LDX was more effective and less costly.

\subsection{Probabilistic Sensitivity Analysis}

The mean probabilistic ICER for the base-case analysis was estimated to be $£ 1586$ per QALY. When assuming an acceptable willingness to pay of $£ 20,000$ per QALY [21], LDX had an $86 \%$ probability of being cost effective when 
Table 3 Base-case analysis results (per patient)

\begin{tabular}{|c|c|c|c|c|c|}
\hline Strategies & Total costs $(\mathfrak{f})$ & Total QALYs & Incremental costs $(\mathfrak{E})$ & Incremental QALYs & ICER (£/QALY) \\
\hline ATX & 2332 & 0.8092 & - & - & - \\
\hline LDX & 2352 & 0.8202 & 19.68 & 0.011 & 1802 \\
\hline
\end{tabular}

$A T X$ atomoxetine, ICER incremental cost-effectiveness ratio, $L D X$ lisdexamfetamine dimesylate, $Q A L Y$ quality-adjusted life-year

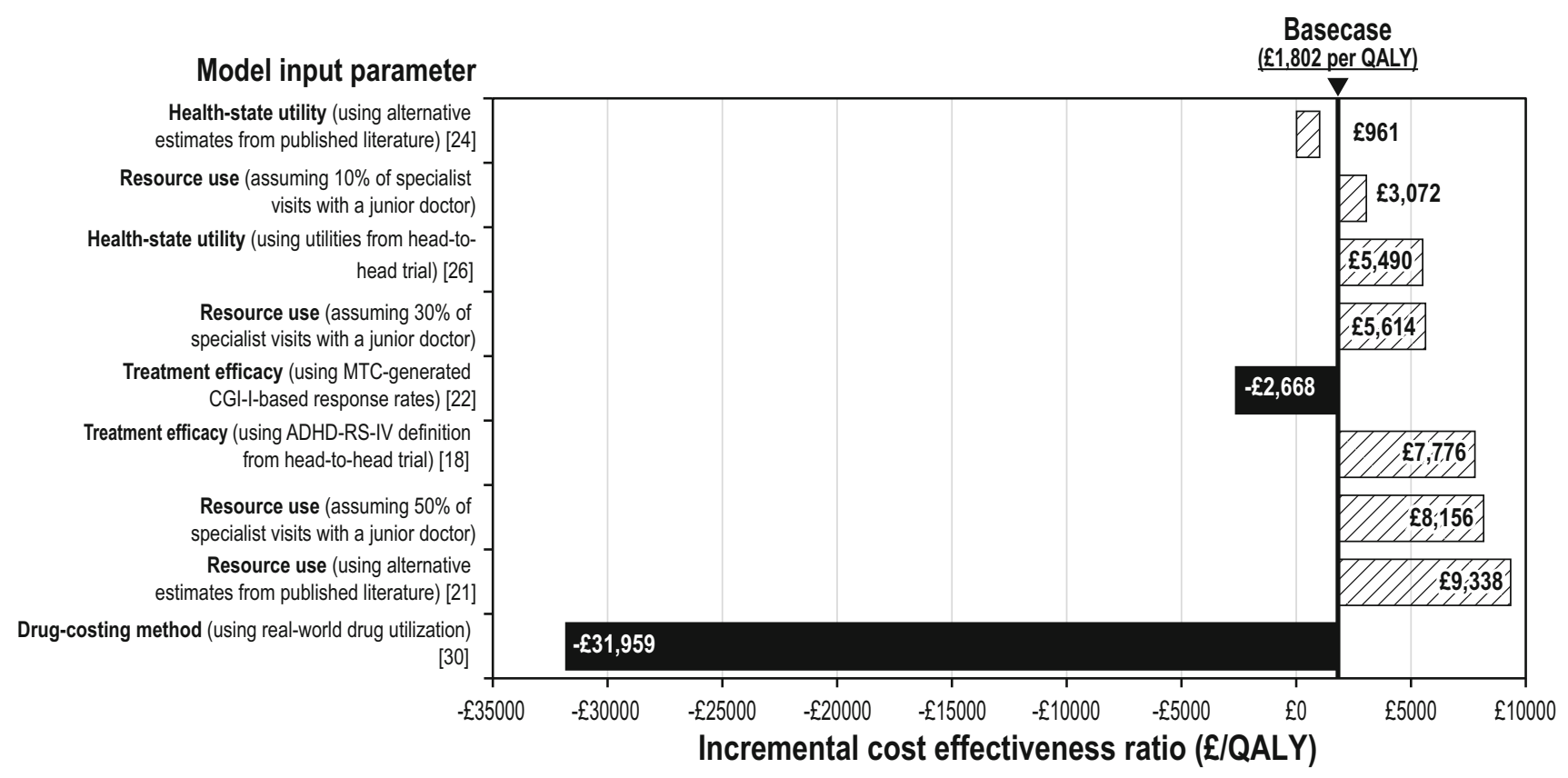

Fig. 2 Summary of sensitivity analysis results. ADHD-RS-IV Attention-Deficit/Hyperactivity Disorder Rating Scale-IV, CGI-I Clinical Global Impression-Improvement subscale, $M T C$ mixed-treatment comparison, $Q A L Y$ quality-adjusted life-year

compared with atomoxetine (Fig. 3). The PSA was also performed for the additional two scenario analyses. For the analysis using alternative efficacy estimates from the MTC, the mean probabilistic ICER showed that LDX was a dominant strategy compared with atomoxetine. For the analysis using utility weight distributions from the head-tohead trial, the mean probabilistic ICER was estimated at $£ 4968$ per QALY, with a $62 \%$ probability that the ICER fell below a threshold value of $£ 20,000$ per QALY.

\section{Discussion}

ADHD in children and adolescents can be successfully treated pharmacologically, and stimulants are the first-line treatment option. However, about one-third of patients have an inadequate response to initial treatment with methylphenidate, and atomoxetine is typically used in this patient population. In a head-to-head, randomized controlled trial, LDX was shown to provide more rapid and superior symptom control than atomoxetine in children and adolescents with an inadequate response to methylphenidate. Our economic analysis used the results of the head-tohead trial to assess the cost effectiveness of LDX when compared with atomoxetine. The base-case results showed that LDX was a cost-effective option when compared with atomoxetine, with an ICER of $£ 1802$ per QALY gained. Moreover, when we used the results of the MTC as clinical inputs, therefore accounting for all available published evidence in this ADHD population, the model results showed that LDX was a dominant strategy. Sensitivity analyses confirmed the robustness of the results, showing a high likelihood of LDX being cost effective relative to atomoxetine when applying probabilistic methods.

Our model has underlying assumptions similar to those of other models used to assess the cost effectiveness of drug therapies for ADHD. The cost effectiveness of atomoxetine in stimulant-exposed UK populations was previously assessed from the NHS perspective by Cottrell et al. [11]. That study showed that inclusion of atomoxetine in the treatment algorithm was cost effective under the established threshold of $£ 20,000$ per QALY in stimulant- 
Table 4 One-way sensitivity analysis results (per patient)

\begin{tabular}{lrcc}
\hline Input parameter & $\begin{array}{l}\text { Incremental } \\
\text { costs }(\mathfrak{f})\end{array}$ & $\begin{array}{l}\text { Incremental } \\
\text { QALYs }\end{array}$ & \begin{tabular}{c} 
ICER (£/QALY) \\
\hline Base case
\end{tabular} \\
Treatment efficacy (using ADHD-RS-IV definition from head-to-head trial) [18] & 49.68 & 0.011 & 1802 \\
Treatment efficacy (using MTC-generated CGI-I-based response rates) [23] & -38.79 & 0.015 & 7776 \\
Health-state utility (using alternative estimates from published literature) [25] & 19.68 & 0.021 & 961 \\
Health-state utility (using utilities from head-to-head trial) [26] & 19.68 & 0.004 & 5490 \\
Resource use (using estimates reported in King et al. [22]) & 102.00 & 0.011 & 9338 \\
$10 \%$ of specialist visits with a junior doctor & 33.56 & 0.011 & 3072 \\
$30 \%$ of specialist visits with a junior doctor & 61.32 & 0.011 & 5614 \\
$50 \%$ of specialist visits with a junior doctor & 89.08 & 0.011 & 8156 \\
Drug-costing method [30] & -349.08 & 0.011 & Dominant: $-31,959$
\end{tabular}

ADHD-RS-IV Attention-Deficit/Hyperactivity Disorder Rating Scale-IV, CGI-I Clinical Global Impression-Improvement subscale, ICER incremental cost-effectiveness ratio, $M T C$ mixed-treatment comparison, $Q A L Y$ quality-adjusted life-year

Fig. 3 Cost-effectiveness acceptability curve. $L D X$ lisdexamfetamine dimesylate, $Q A L Y$ quality-adjusted life-year

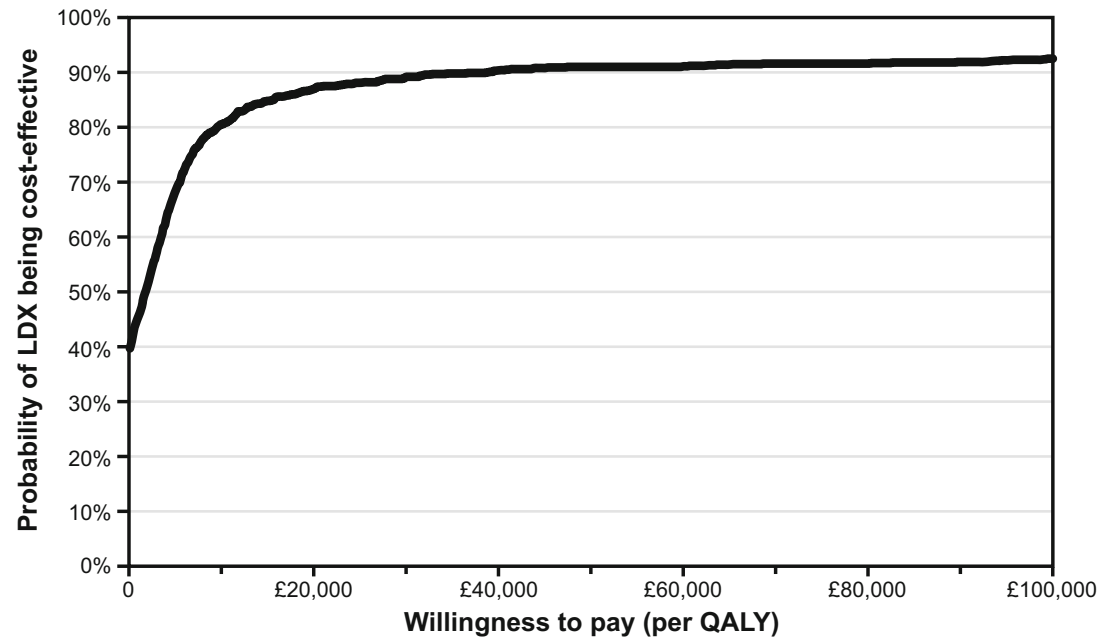

failed or stimulant-contraindicated populations. NICE's current clinical recommendations for the management of ADHD in children and adolescents are based on a costeffectiveness analysis assessing methylphenidate, atomoxetine, and dexamphetamine [22]. The optimal treatment strategy identified by that analysis suggested that methylphenidate should be used in first line, dexamphetamine in second line, with atomoxetine in third line. It also concluded that, for patients contraindicated to stimulants, atomoxetine is preferred to no treatment.

Our model found that LDX is highly cost effective relative to atomoxetine, providing better efficacy at a small increased cost. Thus, LDX would provide a good additional second-line stimulant option. The option of using LDX may be particularly valuable for patients for whom atomoxetine is contraindicated or for those patients who prefer a stimulant with an adverse-event profile that is similar to that of other established stimulants. Thus, the inclusion of a new cost-effective option in the armamentarium of ADHD treatments could allow for patients' individual needs and preferences to be taken into consideration. Having multiple stimulant and nonstimulant treatment options available is likely to contribute to a reduction in the overall burden of ADHD.

The previous HTA economic evaluation [22] acknowledged the lack of clinical evidence to fully assess differences between various ADHD treatments. In contrast, our economic analysis used evidence generated by a randomized, head-to-head controlled trial that is considered to be at the top of the hierarchy of evidence [21,34], although we acknowledge that certain elements of the study design (the 9-week duration and once-daily dosing regimen) may not have elicited the maximum potential treatment benefit of atomoxetine [15]. To account for this, we performed a 
sensitivity analysis using the results of an MTC where all clinical trials of atomoxetine were included, including those with different durations and dosing patterns. The results using the outputs of the MTC were more favorable for LDX than those using the results of the clinical trial. Another strength of our analysis is that our model used a model structure and timeframe of 1 year. This timeframe is consistent with that used in other UK-based models $[11,22,35]$ and requires minimal extrapolation of the short-term data from the head-to-head trial, thus recognizing the lack of the long-term data that would be necessary to adequately inform a longer-term model.

Our study has a number of limitations that need to be considered, particularly when assessing its generalizability. However, to avoid speculation, the effects of these limitations on the model outcomes are not fully explored, given that relevant data are not available to enable these alternative analyses. First, the study was conducted from the UK NHS perspective and did not include the broader societal perspective, which is an important cost driver in the overall cost burden of ADHD. Taking into account costs associated with the societal perspective would likely result in a lower ICER for LDX. Second, the clinical data used in the analysis were derived from a single clinical trial set mainly outside of the UK. However, the European Medicines Agency considered these clinical data to be applicable to all European Union countries. Third, although this trial's strength was in its head-to-head randomized, double-blind, parallel-group, dose-optimized design, the 9-week duration might not have elicited the maximum potential treatment benefit of atomoxetine as it has been suggested that 12 weeks is needed for atomoxetine to reach maximum response [20]. Fourth, despite evidence to suggest that LDX provides better adherence and persistence than atomoxetine [36, 37], the model did not consider real-life medication adherence because of the lack of data on the relationship between adherence to therapy and symptom reduction. Fifth, because of a lack of data, the study did not consider any treatment for nonresponders and assumed these patients discontinued drug therapy. Sixth, the utility data for intolerable side effects were not based on disutility data of individual side effects leading to discontinuation, but estimated in the model as a 50/50 \% mix of the responder and nonresponder utility values. In real-world clinical practice, such patients may receive off-label medications (such as bupropion, clonidine, modafinil, and imipramine) or combination treatments [8]. Seventh, cost effectiveness of any treatment can change over time as clinical practice and medication pricing evolves. Finally, the comparisons that could be performed in this economic analysis were restricted to atomoxetine only because of a lack of data on other possible comparators, such as dexamphetamine.

\section{Conclusions}

The findings from this study support the use of LDX in the UK as a more cost-effective treatment option than atomoxetine in the treatment of children and adolescents who have experienced unsatisfactory improvements on methylphenidate.

Acknowledgments The authors would like to thank Dr. David Coghill, a paid consultant to RTI Health Solutions, who provided clinical advice, and Neil Roskell, who conducted the mixed-treatment analysis of the ADHD trials, funded by Shire Development LLC. Proofreading of the manuscript, and coordination and collation of comments were provided by Caudex, Oxford, UK (funded by Shire International GmbH, Switzerland). Stephen M. Beard, Paul Hodgkins, Matthias Bischof, Josephine A. Mauskopf, and Juliana Setyawan contributed equally to this work. Although employees of Shire were involved in the design, collection, analysis, interpretation, and fact checking of information, the content of this manuscript, the interpretation of the data, and the decision to submit the manuscript for publication in CNS Drugs was made by the authors independently.

Author contributions All authors contributed to the study design and coordination. EAZ led model development and performed economic analyses; JS, an employee of shire at the time of the study, critically reviewed the model and the economic analyses, SMB led the programming of the model; $\mathrm{MB}$, an employee of Shire, contributed to the design of the ADHD resource use study and critically reviewed the economic analyses; $\mathrm{PH}$, an employee of Shire at the time the model was developed, provided overall direction to the model development; JAM assisted with the model design and interpretation of the results and contributed to the preparation of the manuscript. JS acted as overall guarantor. All authors reviewed and approved the final version of the manuscript for publication.

\section{Compliance with Ethical Standards}

Ethical standards This was a non-interventional cost-utility analysis; therefore, institutional review board approval and informed consent were deemed unnecessary. This article does not contain any studies with human participants/animals performed by any of the authors.

Funding This study was funded by Shire Development LLC. Funding was provided to RTI Health Solutions to develop the model. Open access was funded by Shire International $\mathrm{GmbH}$.

Conflict of interest EAZ and JAM are employees of RTI Health Solutions. MB is an employee of Shire. PH and JS were employees of Shire when this work was conducted. SMB was an employee of RTI when this work was conducted and is now employed by BresMed Health Solutions, Sheffield, UK.

Open Access This article is distributed under the terms of the Creative Commons Attribution-NonCommercial 4.0 International License (http://creativecommons.org/licenses/by-nc/4.0/), which permits any noncommercial use, distribution, and reproduction in any medium, provided you give appropriate credit to the original author(s) and the source, provide a link to the Creative Commons license, and indicate if changes were made. 


\section{References}

1. Polanczyk G, de Lima MS, Horta BL, Biederman J, Rohde LA. The worldwide prevalence of ADHD: a systematic review and meta-regression analysis. Am J Psychiatry. 2007;164:942-8.

2. Thomson A, Maltezos S, Paliokosta E, Xenitidis K. Amfetamine for attention deficit hyperactivity disorder in people with intellectual disabilities. Cochrane Database Syst Rev. 2009;1: CD007009.

3. American Psychiatric Association. Diagnostic and statistical manual of mental disorders. 5th ed. Washington, DC: American Psychiatric Association; 2013.

4. Harpin V. The effect of ADHD on the life of an individual, their family, and community from preschool to adult life. Arch Dis Child. 2005;90(Suppl 1):i2-7.

5. Danckaerts M, Sonuga-Barke EJ, Banaschewski T, Buitelaar J, Döpfner M, Hollis C, et al. The quality of life of children with attention deficit/hyperactivity disorder: a systematic review. Eur Child Adolesc Psychiatry. 2010;19(2):83-105.

6. Doshi JA, Hodgkins P, Kahle J, Sikirica V, Cangelosi MJ, Setyawan $\mathrm{J}$, et al. Economic impact of childhood and adult attention-deficit/hyperactivity disorder in the United States. J Am Acad Child Adolesc Psychiatry. 2012;51(10):990-1002.

7. Le HH, Hodgkins P, Postma MJ, Kahle J, Sikirica V, Setyawan J, et al. Economic impact of childhood/adolescent ADHD in a European setting: the Netherlands as a reference case. Eur Child Adolesc Psychiatry. 2014;23(7):587-98.

8. National Institute for Health and Care Excellence. Attention deficit hyperactivity disorder: diagnosis and management of ADHD in children, young people and adults. NICE Clinical Guideline 72. Manchester, UK: National Institute for Health and Care Excellence; 2008. http://www.nice.org.uk/guidance/cg72. Accessed 11 Nov 2014.

9. Childress AC, Sallee FR. Attention-deficit/hyperactivity disorder with inadequate response to stimulants: approaches to management. CNS Drugs. 2014;28(2):121-9.

10. Banaschewski T, Coghill D, Santosh P, Zuddas A, Asherson P, Buitelaar J, et al. Long-acting medications for the hyperkinetic disorders: a systematic review and European treatment guideline. Eur Child Adolesc Psychiatry. 2006;15:476-95.

11. Cottrell S, Tilden D, Robinson P, Bae J, Arellano J, Edgell E, et al. A modeled economic evaluation comparing atomoxetine with stimulant therapy in the treatment of children with attentiondeficit/hyperactivity disorder in the United Kingdom. Value Health. 2008;11:376-88.

12. Coghill D, Banaschewski T, Lecendreux M, Soutullo C, Johnson M, Zuddas A, et al. European, randomized, phase 3 study of lisdexamfetamine dimesylate in children and adolescents with attention-deficit/hyperactivity disorder. Eur Neuropsychopharmacol. 2013;23(10):1208-18.

13. Findling RL, Childress AC, Cutler AJ, Gasior M, Hamdani M, Ferreira-Cornwell MC, et al. Efficacy and safety of lisdexamfetamine dimesylate in adolescents with attention-deficit/hyperactivity disorder. J Am Acad Child Adolesc Psychiatry. 2011;50:395-405.

14. Biederman J, Krishnan S, Zhang Y, McGough JJ, Findling RL. Efficacy and tolerability of lisdexamfetamine dimesylate (NRP104) in children with attention-deficit/hyperactivity disorder: a phase III, multicenter, randomized, double-blind, forced-dose, parallel-group study. Clin Ther. 2007;29:450-63.

15. Dittmann RW, Cardo E, Nagy P, Anderson CS, Bloomfield R, Caballero B. A head-to-head, randomized, double-blind, phase 3b study comparing the efficacy and safety of lisdexamfetamine dimesylate and atomoxetine in the treatment of attention-deficit/ hyperactivity disorder. CNS Drugs. 2013;27(12):1081-92.
16. Guy W. ECDEU assessment manual for psychopharmacology. Rockville: US Department of Health, Education, and Welfare, Public Health Service, Alcohol, Drug Abuse, and Mental Health Administration, NIMH, Psychopharmacology Research Branch Division of Extramural Research Programs; 1976. p. 218-22.

17. DuPaul GJ, Power T, Anastopoulos A, Reid R. The ADHD Rating Scale-IV, checklist, norms, and clinical interpretation. New York: Guildford Press; 1998.

18. Statistical analysis of utility data reported in study SPD489-317. Data on file. Shire Pharmaceuticals; 2013.

19. American Academy of Pediatrics. ADHD: clinical practice guideline for the diagnosis, evaluation, and treatment of attention deficit/hyperactivity disorder in children and adolescents. Process of care supplemental appendix. Pediatrics. 2011a;128:SI1-21.

20. Wilens TE, Newcorn JH, Kratochvil CJ, Gao H, Thomason CK, Rogers $\mathrm{AK}$, et al. Long-term atomoxetine treatment in adolescents with attention-deficit/hyperactivity disorder. J Pediatr. 2006;149(1):112-9.

21. National Institute for Health and Care Excellence. Process and methods guides. Guide to the methods of technology appraisal 2013. Manchester, UK: National Institute for Health and Care Excellence; 2013. http://publications.nice.org.uk/pmg9. Accessed 11 Nov 2014.

22. King S, Griffin S, Hodges Z, Weatherly H, Asseburg C, Richardson $\mathrm{G}$, et al. A systematic review and economic model of the effectiveness and cost-effectiveness of methylphenidate, dexamfetamine and atomoxetine for the treatment of attention deficit hyperactivity disorder in children and adolescents. Health Technol Assess. 2006;10:iii-iv, xiii-146.

23. Roskell NS, Setyawan J, Zimovetz EA, Hodgkins P. Systematic evidence synthesis of treatments for ADHD in children and adolescents: indirect treatment comparisons of lisdexamfetamine with methylphenidate and atomoxetine. Curr Med Res Opin. 2014;30(8):1673-85.

24. Coghill D, Spender Q, Barton J, et al. Measuring quality of life in children with attention-deficit-hyperactivity-disorder in the United Kingdom. Poster P-015-240. Proceedings of the 16th World Congress of the International Association of Child and Adolescent Psychiatry and Allied Professions, Berlin, Germany, August 2004, p. 327-28.

25. Lloyd A, Hodgkins P, Sasane R, Akehurst R, Sonuga-Barke EJ, Fitzgerald $\mathrm{P}$, et al. Estimation of utilities in attention-deficit hyperactivity disorder for economic evaluations. Patient. 2011;4(4):247-57.

26. Clinical study SPD489-317 report: a phase 3b, double-blind, randomised, active-controlled, parallel-group study to compare the time to response of lisdexamfetamine dimesylate to atomoxetine hydrochloride in children and adolescents aged 6-17 years with attention-deficit/hyperactivity disorder (ADHD) who have had an inadequate response to methylphenidate therapy. Data on file. Shire Pharmaceuticals; 2013.

27. ADHD resource study (UK). MEDIX Intelligent Information: market research report. Data on file. Shire Pharmaceuticals; 2012.

28. Department of Health. Reference costs 2011-12. London: Department of Health; 2012. http://www.dh.gov.uk/health/2012/ 11/2011-12-reference-costs/. Accessed 11 Nov 2014.

29. British National Formulary No 64. London: British National Formulary; 2012. http://www.medicinescomplete.com/mc/bnf/ current/. Accessed 1 Sept 2012.

30. IMS prescription panel data. Data on file. Shire Pharmaceuticals; 2011.

31. Zimovetz EA, Setyawan J, Beard SM, Hodgkins P. Systematic review of health-state utilities in attention deficit hyperactivity disorder. Value Health. 2012;15:A334. Poster PMH5. Proceedings of the 15th Annual European Congress of the International 
Society for Pharmacoeconomics and Outcomes Research, Berlin, Germany, November 2012.

32. Curtis L. Unit costs of health and social care 2011. Canterbury: Personal Social Services Research Unit, University of Kent; 2012.

33. Horsman J, Furlong W, Feeny D, Torrance G. The Health Utilities Index (HUI): concepts, measurement properties and applications. Health Qual Life Outcomes. 2003;1:54.

34. Teutsch SM, Berger ML, Weinstein MC. Comparative effectiveness: asking the right questions, choosing the right method. Health Aff (Millwood). 2005;24(1):128-32.

35. British Psychological Society, Royal College of Psychiatrists. Attention deficit hyperactivity disorder: the NICE guideline on diagnosis and management of ADHD in children, young people, and adults. National Practice Guideline Number 72. Leicester, UK: British Psychological Society, and London: Royal College of Psychiatrists; 2009. http://www.ncbi.nlm.nih.gov/books/ NBK53652/. Accessed 28 Nov 2014.

36. Setyawan J, Hodgkins P, Guérin A, Gauthier G, Cloutier M, Wu $\mathrm{EQ}$, et al. Comparing treatment adherence of lisdexamfetamine and other medications for the treatment of attention deficit/hyperactivity disorder: a retrospective analysis. J Med Econ. 2013;16(7):962-75.

37. Setyawan J, Guérin A, Hodgkins P, Gauthier G, Cloutier M, Wu $\mathrm{E}$, et al. Treatment persistence in attention deficit/hyperactivity disorder: a retrospective analysis of patients initiated on lisdexamfetamine vs other medications. J Med Econ. 2013;16(11): $1275-89$. 